\title{
On the nonexistence of Hausdorff-like metrics for fuzzy sets
}

\author{
Peter Brass* \\ Institut für Informatik, FU Berlin \\ Takustrasse 9, D-14195 Berlin, Germany
}

\begin{abstract}
There has been a number of papers proposing different extensions of the Hausdorff metric to fuzzy sets. None of these proposals behaves as one would intuitively expect. It is the aim of this paper to show that under a reasonable set of axioms, like triangle inequality, invariance under motions, and independence of the length unit, there is no metric on the nonempty bounded fuzzy subsets of a euclidean space.
\end{abstract}

\section{Introduction}

For almost any set of interesting objects it is of fundamental importance to have a metric on them, so that if two objects are not the same, one can decide whether they differ much, or are near to each other. For bounded sets in euclidean spaces the most important metric is the Hausdorff metric:

$$
d_{\text {Hausdorff }}(X, Y):=\max \left(\sup _{x \in X} \inf _{y \in Y} d_{\text {euclidean }}(x, y), \sup _{y \in Y} \inf _{x \in X} d_{\text {euclidean }}(x, y)\right) .
$$

Thus the Hausdorff metric is way to extend a given distance measure for points (in this case the euclidean metric) to a distance measure for (bounded) sets of points (for other set distance measures see Gruber 1993).

A further extension of the metric from sets of points to fuzzy sets has been attempted a number of times. A fuzzy set is a set in which the points have weights from $[0,1]$, where weight 0 means the point is not contained in the set, and weight 1 means the point is completely contained in the set. Thus it is just an extension of the possible values of the indicator function of a set $S$ from $\{0,1\}$ to $[0,1]$. A fuzzy set which is also a classical set (all weights either 0 or 1 ) is called a 'crisp' set.

These proposed extensions of the Hausdorff metric from classical to fuzzy sets always turned out to behave in some ways counterintuitive and unsatisfactory. The first proposal (Dubois, Prade 1983) was not even a metric; the second (Chaudhuri, Rosenfeld 1996) was defined only for the class of sets reaching some given maximum density (e.g. those sets which contain some 'crisp' points), the third and fourth (Chaudhuri, Rosenfeld 1996) are behaving discontinuously under weight changes (if there are several points of maximum weight, and one of them changes its weight), with the third additionally failing to distinguish some fuzzy sets differing in the weights only, and the fourth additionally failing to respect scaling of the underlying distance. The fifth proposal (Boxer 1997) introduces an additional 'crisp' set which is added to every set, so the resulting metric does not even agree with the Hausdorff metric on 'crisp' sets (not even on one-point sets), also it

* e-mail brass@inf.fu-berlin.de 
loses the motion invariance and introduces a strong element of arbitrariness by the choice of that additional set. Furthermore it requires that one restricts the underlying space, since it assumes that the set of points of weight zero (normally almost the whole euclidean space) is compact. The sixth proposal (Fan 1998) is the most promising so far, a different variant of the second, avoiding the 'problem of the empty level sets' by clipping the Hausdorff distance at some given maximum distance $c\left(d_{\text {new }}(X, Y)=\min \left(c, d_{\text {Hausdorff }}(X, Y)\right)\right.$. Thus it has an artificial distance bound $c$, so it does not respect scaling of the underlying set, does not agree with the Hausdorff distance for 'crisp' sets with distance bigger than $c$, and involves choice of the arbitrary constant $c$.

It is the aim of this note to show that these difficulties are in fact unavoidable: there is no good definition of Hausdorff metric for fuzzy sets. A reasonable set of properties we would expect of the distance measure really has no solution.

\section{The impossibility result}

We will study metrics that are defined on all bounded, nonempty fuzzy subsets of an euclidean space (where nonempty means they contain at least one point of positive weight, and bounded means that the diameter of the set of points of positive weight is bounded). It is important that the metric is really defined on all such sets, the second proposal shows that restricting the domain of the metric does make an important difference. In the following we will use 'set' as short version of 'nonempty, bounded fuzzy set'. We believe that it is natural to demand of our metric $d$ the following properties:

[0] positivity: $0 \leq d(X, Y)<\infty$, with $d(X, Y)=0$ if and only if $X=Y$.

[1] triangle inequality: $d(X, Z) \leq d(X, Y)+d(Y, Z)$.

[2] motion invariance: If $\mu$ is a rigid motion of the underlying set, then $d(\mu(X), \mu(Y))=$ $d(X, Y)$.

[3] independence of the length unit: If $\sigma_{\lambda}$ is a scaling of the underlying set, changing all distances by the factor $\lambda>0$, then $d\left(\sigma_{\lambda}(X), \sigma_{\lambda}(Y)\right)=\lambda d(X, Y)$.

These axioms seem to be quite inconspicious, they only state that $d$ is a metric which respects the symmetry of the underlying space, and that it scales well: if we use a different length unit in the underlying space, then the set distance $d$ changes by the same factor. Theorem: There is no metric on the nonempty bounded fuzzy sets that satisfies the axioms $0-3$.

\section{Proof of the Theorem}

Let $d$ be a distance on the nonempty bounded fuzzy sets that satisfies axioms $0-3$. Let $f\left(w_{x}, w_{y}, \delta\right)=d(\{x\},\{y\})$, where $x$ and $y$ are points with weight $w_{x}$ and $w_{y}$ that have the euclidean distance $\delta$. By axiom 2 this function $f$ is well-defined, since any different pair $x^{\prime}, y^{\prime}$ is congruent by a rigid motion with $x, y$, and gives the same distance. For the same reason $f$ is symmetric in the weights: $f\left(w_{x}, w_{y}, \delta\right)=f\left(w_{y}, w_{x}, \delta\right)$.

By axiom 3 we have $f\left(w_{x}, w_{y}, \delta\right)=\delta g\left(w_{x}, w_{y}\right)$, where $g\left(w_{x}, w_{y}\right):=f\left(w_{x}, w_{y}, 1\right)$. Now the triangle inequality, applied to three collinear points $x, y, z$ with weights $w_{x}, w_{y}, w_{z}$, and

euclidean distances $d_{\text {euclidean }}(x, y)=\delta_{1}, d_{\text {euclidean }}(y, z)=\delta_{2}$ and $d_{\text {euclidean }}(x, z)=\delta_{1}+\delta_{2}$, gives $f\left(w_{x}, w_{z}, \delta_{1}+\delta_{2}\right) \leq f\left(w_{x}, w_{y}, \delta_{1}\right)+f\left(w_{y}, w_{z}, \delta_{2}\right)$. Thus

$$
g\left(w_{x}, w_{z}\right) \leq \frac{\delta_{1}}{\delta_{1}+\delta_{2}} g\left(w_{x}, w_{y}\right)+\frac{\delta_{2}}{\delta_{1}+\delta_{2}} g\left(w_{y}, w_{z}\right)
$$


and this holds for all $\delta_{1}, \delta_{2}>0$ and $\left.\left.w_{x}, w_{y}, w_{z} \in\right] 0,1\right]$. Now if $g\left(w_{x}, w_{y}\right)>g\left(w_{y}, w_{z}\right)$ we take the limit $\delta_{1} \rightarrow 0$ with $\delta_{2}=1$, else we take the limit $\delta_{2} \rightarrow 0$ with $\delta_{1}=1$. By this we obtain

$$
\left.\left.g\left(w_{x}, w_{z}\right) \leq \min \left(g\left(w_{x}, w_{y}\right), g\left(w_{y}, w_{z}\right)\right) \quad \text { for all } w_{x}, w_{y}, w_{z} \in\right] 0,1\right] .
$$

This implies that the function $g$ is constant. For if there are values $w_{1}, \ldots, w_{4}$ such that $g\left(w_{1}, w_{2}\right) \neq g\left(w_{3}, w_{4}\right)$, then at least one of $g\left(w_{1}, w_{2}\right) \neq g\left(w_{1}, w_{3}\right)$ or $g\left(w_{3}, w_{1}\right) \neq g\left(w_{3}, w_{4}\right)$ holds. We may assume that $g\left(w_{1}, w_{2}\right)<g\left(w_{1}, w_{3}\right)$, then

$$
g\left(w_{1}, w_{3}\right) \leq \min \left(g\left(w_{1}, w_{2}\right), g\left(w_{2}, w_{2}\right)\right) \leq g\left(w_{1}, w_{2}\right)<g\left(w_{1}, w_{3}\right),
$$

a contradiction. So $g\left(w_{x}, w_{y}\right)=: \gamma$, a constant independent of $w_{x}, w_{y}$. Thus we have for any distinct points $x, y d(\{x\},\{y\})=\gamma d_{\text {euclidean }}(x, y)$ independent of the (positive) weights of $x$ and $y$.

But if $d$ is a metric, then it must give a positive distance for two fuzzy sets consisting of the same point, but with different weights. Let $X_{1}, X_{2}$ be sets consisting of the same point $x$, with weight $\frac{1}{3}$ in $X_{1}$ and weight $\frac{2}{3}$ in $X_{2}$, and let $\varepsilon:=d\left(X_{1}, X_{2}\right)>0$. Take a further set $Y$ consisting of a point $y$ at distance $\frac{\varepsilon}{3 \gamma}$ to $x$, and with an arbitrary positive weight. Then

$$
\varepsilon=d\left(X_{1}, X_{2}\right) \leq d\left(X_{1}, Y\right)+d\left(Y, X_{2}\right)=\gamma d_{\text {euclidean }}(x, y)+\gamma d_{\text {euclidean }}(y, x)=\frac{2}{3} \varepsilon,
$$

a contradiction. This proves our theorem.

\section{Further remarks}

We have shown that there is no metric satisfying the axioms $0-3$ above, so any metric will behave in some way counterintuitive. But of course it is still reasonable to ask for a metric on the fuzzy (bounded nonempty) sets. We believe that axioms 0-2 are really so natural that they should not be violated, which is indeed satisfied by several of the proposals. The theorem shows that we cannot avoid having 'special' lengths, essentially because we are trying to measure to incompatible things at the same time: a geometric distance (for which the invariance of the length unit is really an unavoidable axiom) and the distance of the density functions. Thus fuzzy sets are really essentially different from normal sets, since for normal sets the Hausdorff distance does satisfy our axioms.

\section{References}

Boxer, L, 1997. On Hausdorff-like metrics for fuzzy sets, Pattern Recognition Letters 18 115-118, see also Erratum, same volume, 505-506.

Chaudhuri, B.B., Rosenfeld, A., 1996. On a metric distance between fuzzy sets, Pattern Recognition Letters 17 1157-1160.

Dubois, D., Prade, H., 1983. On distances between fuzzy sets and their use for plausible reasoning, in: Proc. Int. Conf. on Systems, Man and Cybernetics 300-303.

Fan, J.-I., 1998. Note on Hausdorff-like metrics for fuzzy sets, Pattern Recognition Letters $19793-796$. 
Gruber, P., 1993. Approximation of convex bodies, 319-345 in Handbook of Convex Geometry Vol A, P.M. Gruber and J.M. Wills, Eds., Elsevier/North Holland.

Rosenfeld, A., 1985. Distances between fuzzy sets, Pattern Recognition Letters 3229 233. 